\title{
Intracellular ion changes induced by the exposure to beta-amyloid can be explained by the formation of channels in the lysosomal membranes
}

\author{
Dmitry V. Zaretsky*, Maria V. Zaretskaia \\ Zarbio, Chapel Hill, NC 27516, USA
}

\begin{abstract}
In this manuscript, we reassess the data on beta-amyloid-induced changes of intracellular ions concentrations published previously by Abramov et al. $(2003,2004)$. Their observations made using high-resolution confocal microscopy with fast temporal resolution of images formed by fluorescent ion-sensitive fluorescent probes in living cells represent an unequivocal support for the amyloid channel theory. However, closer look reveals multiple facts which cannot be explained by channel formation in plasma membrane. Recently proposed amyloid degradation toxicity hypothesis provides the interpretation to these facts by considering that channels are formed in the lysosomal membranes.
\end{abstract}

Keywords: beta-amyloid toxicity, proteolytic digestion, membrane channel, intracellular ion disturbances, calcium homeostasis, intracellular $\mathrm{pH}$, lysosome.

\section{Amyloid channels and their visual demonstration using high-resolution microscopy}

The amyloid channel theory of Alzheimer's disease is the only amyloid-centric theory that identifies the primary molecular interaction which can be directly linked to the processes resulting in neuronal disfunction and eventual cell death. In the 1990s, several groups used electrophysiological techniques to demonstrate the formation of ion channels in lipid membranes by either $A \beta_{1-40}$, or $A \beta_{1-42}$, or its short fragment $\left(A \beta_{25-35}\right)[4-7,10,19,22-25]$. Atomic force microscopy images of beta-amyloid aggregates [18] supported the existence of such channels. However, AFM studies were performed in artificial membranes, while it was critical to demonstrate unequivocally that amyloid-induced ion disturbances in living cells can be attributed to membrane channels.

The studies by Abramov et al. [1, 2] provided the most visual support for the amyloid channel theory. Along with numerical data, the authors described and showed the convincing visual of intracellular ion concentrations, which at first glance is impossible to interpret in any other way than a formation of the nonselective amyloid channels in the plasma membrane. The theory of the methods for measuring intracellular concentrations of ions is described in the Supplemental materials for the reference. The cited publications are unique in that they contain not one but several key observations which are critical for the support of the amyloid channel theory.

First, the studies confirmed that the exposure to beta-amyloid increased intracellular calcium levels. However, the authors stressed that the responses were not smooth changes recorded in some studies [9], but consisted of sporadic fluctuations in cytoplasmic calcium similar to the observations of others [8]. These fluctuations looked like waves or oscillations running over minutes, so they occur over a much slower time scale compared to typical calcium changes occurring in these non-excitable cells. Also, when manganese is present in the incubation medium, each increase of calcium is accompanied by a proportionate entry of manganese, which matches the concept of channel non-selectivity.

Next, using confocal imaging with a high temporal and spatial resolution, the authors demonstrated that increased concentrations of cytoplasmic calcium looked as if they were generated primarily by diffusion from a local source. The spike originated from a focal point and spread through the cell. However, unlike self-propagating IP3 based signals, calcium waves were decrementing with distance.

Consistent with most studies on cells, the formation of channels was not immediate. Calcium waves started to appear at least several minutes after an addition of the peptide to the incubation medium.

Finally, the concentrations of cytoplasmic calcium were mirrored by intracellular concentrations of other ions: sodium, potassium, and protons. Similarly, each wave of calcium corresponds to the inflow of manganese. It appeared that once the membrane becomes permeable to one ion, other ions start flowing across the membrane as well.

These observations together match the concept of membrane channel formation well. Nevertheless, there were some discrepancies between the observed ion fluxes and those which can be predicted based on existing ion gradients. 

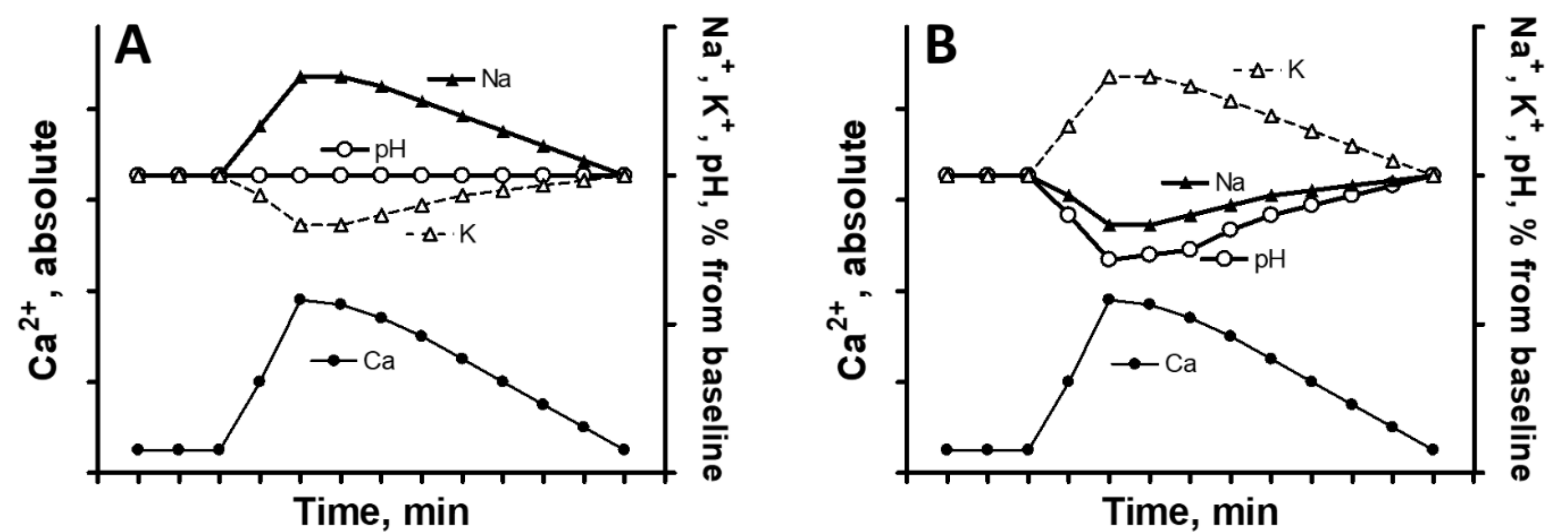

Figure 1. Schematics of the changes of intracellular ion concentrations induced by $A \beta$ exposure.

A: Theoretical prediction based on the permeabilization of plasma membrane.

B: Experimentally observed changes.

lon changes do not match theoretical predictions based on channel formation in plasma membranes

The focal increase in the concentration, which extends over time and decreases with distance, is so visual that it is very difficult to reject that the membrane permeabilization occurs very locally in a specific spot. This local disruption of the barrier function does not spread as it would happen with a lipid vesicle if it was broken by a physical object. Instead, it is quickly repaired. Also, as was shown in electrophysiological studies, the channels permeabilize membranes to calcium, sodium, potassium, and other ions. The fact that intracellular ion concentrations mirror each other is consistent with the non-selectivity of channels. We cannot find arguments to reject channel formation as the reason of ion disturbances. However, a closer look reveals multiple facts that cannot be explained by channel formation in plasma membrane. The key word here is "plasma".

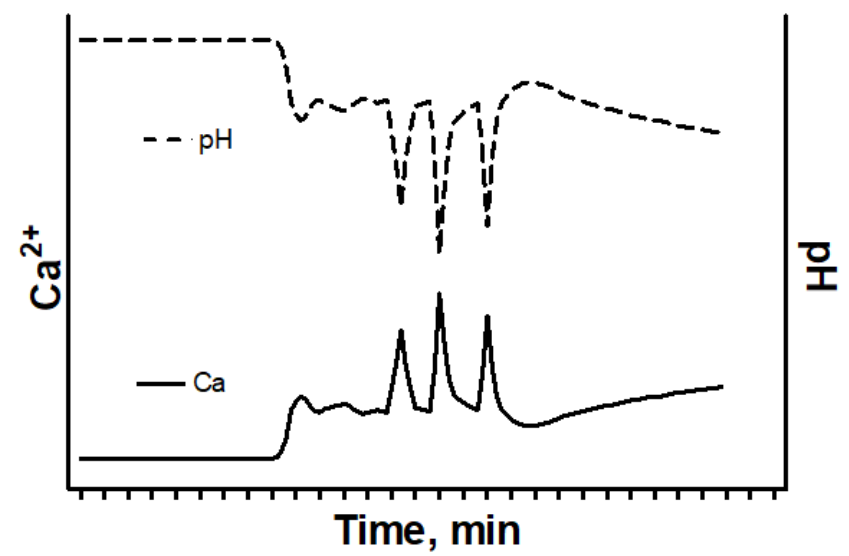

Figure 2. The schematic showing experimental changes of intracellular calcium concentration waves coincident with oscillations of intracellular $\mathrm{pH}$ observed after exposure to $A \beta$.
First, while raising the intracellular calcium matches the concept of a plasma membrane channel, concentrations of other ions mirroring the calcium fluctuations are not consistent with common sense predictions based on existing transmembrane gradients. It is typical for the cytoplasm to have sodium levels below that of the extracellular liquid, while the intracellular potassium concentration is higher than the one in cellular surroundings. In this case, opening the plasma membrane channels should increase intracellular sodium, but decrease intracellular potassium (Fig.1A). However, in the cited manuscripts, Abramov et al. report exactly the opposite: sodium goes down, while potassium increases (Fig.1B). Non-specific channels in plasma membrane cannot explain the observed changes of ion concentrations.

Second, the calcium influx coincides with acidification waves (Fig.2). The authors suggested that this can be explained by $\mathrm{Ca}^{2+} / \mathrm{H}^{+}$exchange [1], but the degree of synchronization of the flows suggests that they are driven by the same mechanism, rather than being caused by one another. The plasma membrane channel cannot be that mechanism.

Third, the reason for the occurrence of ion concentration oscillations is not known. If we are to accept the concept that the outbound change in concentration is caused by the opening of the channel, then it would be critical to understand what causes the restoration of the concentration. At first glance, it is possible that membrane leakage can be counteracted by active transmembrane transport. However, we argue that the recovery is initiated by the inability of the channel to deliver more ions. Specifically, manganese, which is entering through the channel, cannot be taken out, so after it enters the cell and quenches the calcium-sensitive probe, it remains intracellularly (Fig.3). If the channel would remain open to the source of manganese, manganese would 


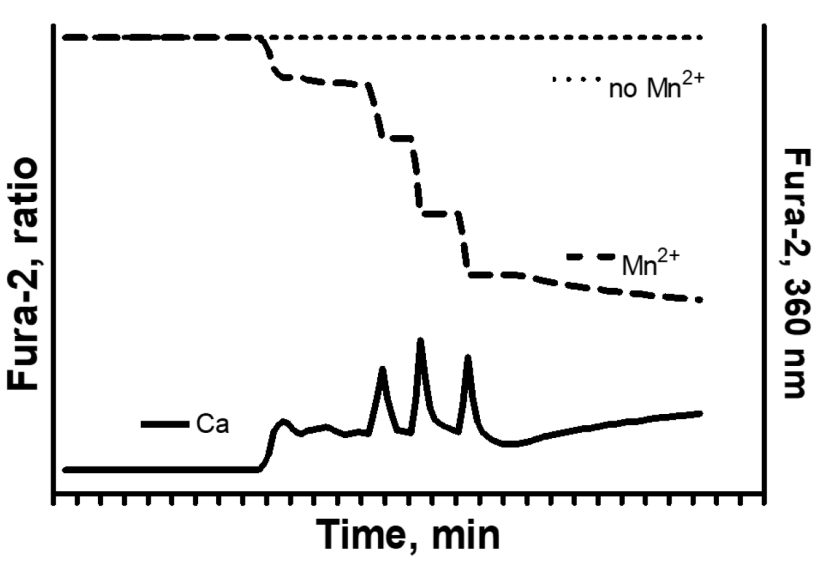

Figure 3. The schematic showing experimental changes of intracellular calcium concentration waves coincident with quenching of Fura-2 fluorescence induced by entering $\mathrm{Mn}^{2+}$ induced by the exposure to $A \beta$.

continue to accumulate intracellularly, but that does not happen. In contrast, other ions, that have active mechanisms defining their intracellular concentrations, such as sodium, potassium, and calcium, return to pre-wave levels.

Quite reasonably, the alternative possibility is that the restoration of ion gradients is associated with a closure of the channel. However, this possibility does not match the frequencies of channel opening-closing recorded electrophysiologically $[4,6,22]$. In model membranes, permeability transitions in amyloid channels occur either in the millisecond range (fast changes) or in a matter of seconds (slower switching). In contrast, waves - recorded in living cells - are on a minute scale. Also, closed channel in model membranes could become reopen again, but that was not readily observable in living cells. An alternative explanation is that the opening of a channel is actually an average of the channel being in an oscillating openclose state. If so, the restoration of ion gradients can be explained by a complete closure of the channel, or more likely its disappearance. This is a reasonable hypothesis, considering that the plasma membrane is continuously repaired. The removal of damaged permeabilized membrane areas is one of the key repairing strategies which any cell, especially an excitable cell, needs to have. However, this second explanation would suggest that such a repair occurs within seconds after the damage takes place: in the original recordings, the moment of channel closure is close to the peak of the wave, which occurs less than 30 seconds after the peak starts (see Fig. 2 in Abramov et al, 2004). In the presented figures, many waves reach their maximum within 10 seconds. We would not claim that such fast repair is not possible, but it feels reasonable to search for an alternative explanation, so we will present one in the next section.
Finally, there are always significant delays in the ion concentration response after beta-amyloid exposure, and the length of these delays has a significant variability. The point of comparison is critical here. In electrophysiological studies, the formation of channels, in fact, takes minutes. In studies on lipid bilayers published by the laboratories of Arispe $[6,7]$ and Kagan [20, 22], the appearance of the current corresponding to the opening of a channel occurs within minutes after the addition of the peptide to the incubation medium. By contrast, in studies, that included lipid vesicles with enclosed fluorescent probes, the permeabilization was fast - Alarcon et al. [3] and Zaretsky et al. [30, 31] reported complete equilibration within seconds after adding the channelforming peptide. This discrepancy is most likely due to the difference between settings - channel-forming units made of peptide can quickly enter the membrane, which happens in liposomes that are efficiently mixed with the added peptide. In turn, the fragility of the lipid bilayer does not allow such fast mixing, so the delay occurs due to the diffusionmediated transport of the peptide to the membrane surface, but not because of slow channel formation itself. From this perspective, the plasma membrane of cells with enclosed fluorescent probe should behave similarly to liposomes, so the channels should form quickly, but this is not what is shown experimentally. As we will discuss below, within our framework of interpretation of molecular and cellular events, this delay of response receives its explanation.

To summarize, while the discussed data supports the amyloid channel theory, there is a list of observations that cannot be or is difficult to explain by channel formation in the plasma membranes:

1. Synchronous and mirroring flows of multiple ions, with the changes in cytoplasmic concentrations of sodium and potassium which are opposite to the potential effects of permeabilization of plasma membranes.

2. Significant intracellular $\mathrm{pH}$ oscillations which mirror calcium waves without any temporal offset.

3. Significant delay in ion responses to betaamyloid exposure.

4. Fast disappearance of calcium inflow and reversal of the cytoplasmic concentrations to prewave level.

5. Presence of multiple short-lived waves that do not correspond to known opening-closing transitions of amyloid channels, which were studied using electrophysiological methods.

\section{Data interpretation based on amyloid degradation toxicity hypothesis}

The recently developed amyloid degradation toxicity hypothesis [32] provides the working framework for interpreting these observations. 
In short, this hypothesis considers that $A \beta$ is taken inside the cells by endocytosis. After the endosome is merged with lysosomes, the peptide is degraded by proteases, which can produce fragments with the ability to form membrane channels. Lysosomal membranes carry a significant negative surface charge, which is needed for the incorporation of the channel into the membrane - so formed channels permeabilize lysosomes. Amyloid channels are not selective, so they can transfer various ions, including sodium, potassium, and calcium [7]. Lysosomal permeabilization was well described previously [16, $28,29]$ and can result in the leakage of even larger compounds [16, 27].

How does the transfer of the location of the action from the plasma membrane to the lysosome fits the described experimental findings of Abramov et al.? We will try to explain the five points mentioned above sequentially.

First, lysosomal permeabilization does not open the exchange between the extracellular space and the cytoplasm, so the predictions about the direction of the ion flows based on concentrations in respective compartments are not relevant anymore. It is important to note that we do not have data on intralysosomal concentrations of sodium and potassium, so we cannot estimate how lysosomal permeabilization would affect cytoplasmic sodium and potassium. Therefore, we cannot assign lysosomal permeabilization as the reason for the observed changes in sodium and potassium. The mirroring of potassium and sodium waves with calcium without temporal offsets allows us to hypothesize that the mechanism is similar between all ions, though.

Second, lysosomal permeabilization readily explains the waves of calcium and counteraction between calcium and protons (see Fig.4 for the illustration). Lysosomes are a well-known storage of calcium, with intralysosomal concentrations exceeding cytoplasmic concentrations by up to two orders [11]. Obviously, the permeabilization of a single lysosome will result in a local spike of calcium concentration (exactly as observed by Abramov et al.). Similarly, intralysosomal content is acidic, with $\mathrm{pH}$ levels below 5 [21], which corresponds to the proton concentration being almost three orders higher than in the cytoplasm. The inflow of protons into the cytoplasm will mirror the inflow of calcium.

Third, along the amyloid degradation toxicity hypothesis, the delay in ion responses after the addition of beta-amyloid to cells is due to the time required for the endocytosis of extracellular amyloid and its digestion into fragments. In fact, endocytosed beta-amyloid accumulates in the lysosomes and remains associated with lysosomal markers for extended periods of time. After uptake, the concentrations of intracellular beta-amyloid fall just below $50 \%$ in $24 \mathrm{~h}$ [28]. Each permeabilized lysosome does not kill the cell; it requires either the cumulative effect of the failure of multiple organelles or the creation of an extremely large membrane channel that releases lysosomal proteases initiating apoptotic and necrotic mechanisms $[14,15,17,26]$. Therefore, this slow time course also explains why the decrease in cell survival requires long incubations (in contrast, large plasma membrane channels should kill exposed cells quickly).

Forth, the influx of calcium into the cytoplasm from a permeabilized lysosome is limited by the storage volume of this lysosome, so the end of the wave will not require any changes to the channel itself - the wave would be short-lived due to the limited amount of calcium stored inside particular lysosome. Importantly, the recovery of pre-wave levels of both calcium and $\mathrm{pH}$ could occur due to the active processes of transmembrane transport controlling the intracellular milieu, but in the case of a single wave, also through existing buffering systems for both ions. Within this framework, if manganese was present in the incubations solution, the ion will be trapped in the endosomes together with endocytosed $A \beta$. Lysosomal permeabilization by non-selective channels would release the trapped manganese and result in stepwise decreases of total fura-2 fluorescence (measured by excitation at $360 \mathrm{~nm}$ ), occurring simultaneously with calcium spikes (measured ratiometrically). Due to the
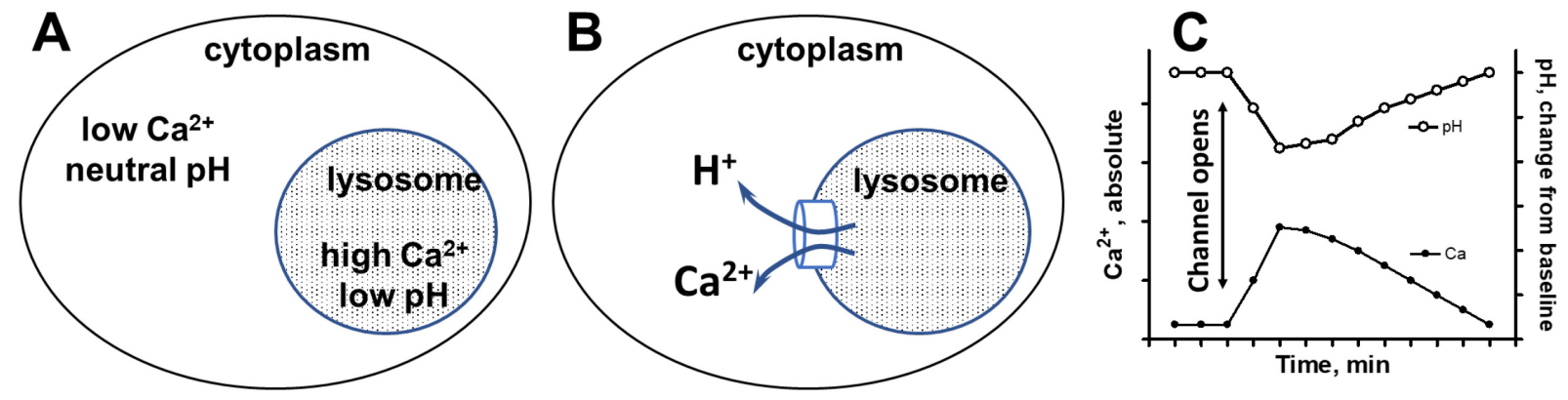

Figure 4. A: Distribution of calcium concentrations and $\mathrm{pH}$ in cytoplasm and lysosomes. B: The direction of ion flows after a channel is formed in lysosomal membrane. C: The changes of cytoplasmic calcium and $\mathrm{pH}$ after lysosomal permeabilization (compare with experimental changes depicted at the Fig. 2 . 
absence of mechanisms that remove manganese from the cytoplasm, this quenching will not look like oscillations or waves, but rather steps.

Finally, the appearance of multiple waves/oscillations in this scenario is most likely the result of the sequential permeabilization of multiple lysosomes inside a single cell. Studies using fluorescently labeled $A \beta$ demonstrate multiple endocytic vesicles that also carry lysosomal markers [28]. Similarly, a fluorescent membrane-impermeant marker such as Lucifer Yellow also accumulates in lysosomes of cultured cells [16]. If cells pre-loaded with Lucifer Yellow are exposed to the exogenous $A \beta$, the leakage of the dye is visible on confocal images $[16,29]$. Importantly, within each cell, it looks like multiple lysosomes become permeabilized, which matches the observation of multiple oscillations of calcium in the discussed studies [1,2].

\section{Conclusions}

Two manuscripts from Abramov et al. provide very visual support of the existence of amyloid channels. For obvious reasons, when cells are exposed to the exogenous amyloid, readers are prone to assume that the target for channel formation is in the plasma membranes of the exposed cell. Due to the incompatibility of this assumption with experimental observations, the results of the outstanding experimental project did not fill the gaps in our knowledge about amyloid toxicity. In this review, we attempted to correct this omission.

\section{Acknowledgements}

The authors thank Daniel Zaretsky for his editorial help.

Research reported in this publication did not receive external funding. 


\section{Supplemental materials}

\section{Fluorometric methods of measuring intracellular ion concentrations}

To interpret the data of Abramov et al. [1, 2] properly, it is important to understand the methods which were used to measure the concentrations of intracellular ions.

Calcium measurements using Fura-2. This fluorescent probe binds calcium with the dissociation constant in the sub-micromolar range [12, 13]. After binding calcium, the absorption maximum of Fura-2 shifts into shorter wavelengths, while the emission maximum remains relatively unchanged. If the emission is measured at $510 \mathrm{~nm}$, then the maximal absorption (and excitation maximum as well) of calcium-free probe occurs at a wavelength of just above $360 \mathrm{~nm}$ and of calcium-bound - at $340 \mathrm{~nm}$. Due to this property, the ratio of fluorescence intensities at maxima corresponding to the free anion form and calcium complex form allows to determine the percent of calcium complex independent of the amount of probe, absolute sensitivity of sensors, etc. To make the ratio most sensitive to the calcium concentration, two fixed wavelengths are used for Fura-2: 340 and $380 \mathrm{~nm}$. Ratiometric probes are preferred for use in cultured cells, where loading with the probe (using membrane-permeable esterified analogs which are converted into non-permeable fluorescent probes by intracellular esterases) can be variable. The spectra of Fura-2 in solutions with various concentrations of free calcium are shown at Fig.A1. This ratio is not dependent on the amount of working probe, so can be used to estimate free calcium concentrations in the presence of quenchers of fluorescence, which make some fura-2 molecules unable to bind calcium.

Fura-2 is charged and is not permeable through undamaged membranes. To load cells with the probe, the neutral cell-permeable acetoxymethyl (AM) ester form (Fura-2AM) is used. After Fura-2AM diffuses

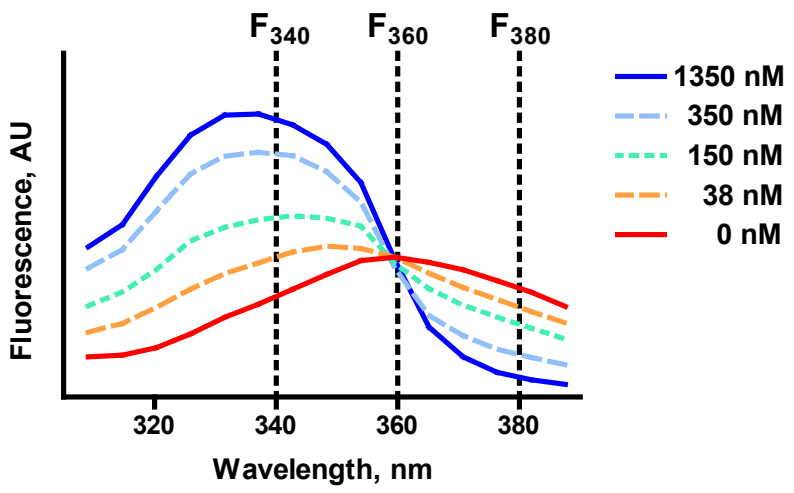

Figure A1. Fluorescence excitation spectra of fura-2 in solutions containing $0-1350 \mathrm{nM}$ free $\mathrm{Ca}^{2+}$. Emission is measured at $510 \mathrm{~nm}$. across the plasma membrane, cytoplasmic esterases cleave AM groups. After enzymatic hydrolysis, the charged compound remains entrapped inside the cell and its fluorescence can be used to measure the cytoplasmic concentration of free calcium (as a ratio $\left.\mathrm{F}_{380} / \mathrm{F}_{340}\right)$.

Due to the unidirectional entry of Fura-2AM into the cells, the intracellular concentration of the probe can significantly exceed the extracellular concentration. By binding calcium, the probe can become a calcium buffer, which would smooth the changes of intracellular calcium. Such smoothing can explain why the oscillatory changes were observed as a uniform increase in some studies.

Quenching of Fura-2 with manganese. If the fluorescence is excited at $360 \mathrm{~nm}$, its intensity does not depend on calcium concentration (isosbestic point) and can be used to estimate the amount of the probe available for the measurements. If manganese $\left(\mathrm{Mn}^{2+}\right)$ is added to quench the fluorescence of Fura-2, the ratiometric measurement is still providing the concentration of free calcium, but the fluorescence excited at $360 \mathrm{~nm}$ can inform about the degree of quenching. The decrease of Fura-2 fluorescence after the addition of $\mathrm{Mn}^{2+}$ can be used as a measure of the quencher availability in the vicinity of the probe. The addition of extracellular manganese does not affect the fluorescence of the intracellular probe until manganese is transferred into the cytoplasm through some mechanism.

Measurement of intracellular $\mathrm{pH}$ and other ions. The principles of measuring $\mathrm{pH}$ using BCECF are the same as those of measuring calcium with Fura-2 - the excitation spectrum of this fluorescein derivative changes over the $\mathrm{pH}$ range that is close to physiological levels. The principles of $\mathrm{pH}$ measurements using SNARF-1 are very similar, except that the spectral shift induced by $\mathrm{pH}$ changes affects the emission spectrum of the fluorescent probe (not excitation as in case of Fura-2 or BCECF). Similarly, SBFI to measure sodium and PBFI to measure potassium concentrations are ratiometric probes (excitation spectra shift after binding corresponding cation).

Unlike Fura-2 and all other used probes, the calcium-sensing probe Fluo-4 does not change the fluorescence spectra after binding calcium. It is essentially non-fluorescent in calcium-free form, but becomes fluorescent after binding calcium. The appearance of fluorescence can be used to monitor free calcium concentrations. Considering the dissociation constant of Fluo-4 with calcium, it can detect increases of calcium concentration below $1 \mu \mathrm{M}$.

All probes are loaded into cells using their respective AM-forms. 


\section{References}

[1] A.Y. Abramov, L. Canevari, M.R. Duchen, Calcium signals induced by amyloid $\beta$ peptide and their consequences in neurons and astrocytes in culture, Biochimica et Biophysica Acta (BBA) - Molecular Cell Research 1742 (2004) 81-87.

[2] A.Y. Abramov, L. Canevari, M.R. Duchen, Changes in intracellular calcium and glutathione in astrocytes as the primary mechanism of amyloid neurotoxicity, The Journal of neuroscience : the official journal of the Society for Neuroscience 23 (2003) 5088-5095.

[3] J.M. Alarcon, J.A. Brito, T. Hermosilla, I. Atwater, D. Mears, E. Rojas, Ion channel formation by Alzheimer's disease amyloid beta-peptide (Abeta40) in unilamellar liposomes is determined by anionic phospholipids, Peptides 27 (2006) 95-104.

[4] N. Arispe, H.B. Pollard, E. Rojas, The ability of amyloid beta-protein [A beta $P$ (1-40)] to form Ca2+ channels provides a mechanism for neuronal death in Alzheimer's disease, Annals of the New York Academy of Sciences 747 (1994) 256-266.

[5] N. Arispe, H.B. Pollard, E. Rojas, beta-Amyloid $\mathrm{Ca}(2+)$-channel hypothesis for neuronal death in Alzheimer disease, Mol Cell Biochem 140 (1994) 119125.

[6] N. Arispe, H.B. Pollard, E. Rojas, Giant multilevel cation channels formed by Alzheimer disease amyloid beta-protein [A beta $\mathrm{P}-(1-40)$ ] in bilayer membranes, Proceedings of the National Academy of Sciences of the United States of America 90 (1993) 10573-10577.

[7] N. Arispe, E. Rojas, H.B. Pollard, Alzheimer disease amyloid beta protein forms calcium channels in bilayer membranes: blockade by tromethamine and aluminum, Proceedings of the National Academy of Sciences of the United States of America 90 (1993) 567-571.

[8] R. Bhatia, H. Lin, R. Lal, Fresh and globular amyloid beta protein (1-42) induces rapid cellular degeneration: evidence for AbetaP channel-mediated cellular toxicity, FASEB journal : official publication of the Federation of American Societies for Experimental Biology 14 (2000) 1233-1243.

[9] A. Demuro, E. Mina, R. Kayed, S.C. Milton, I. Parker, C.G. Glabe, Calcium Dysregulation and Membrane Disruption as a Ubiquitous Neurotoxic Mechanism of Soluble Amyloid Oligomers, Journal of Biological Chemistry 280 (2005) 17294-17300.

[10] S.R. Durell, H.R. Guy, N. Arispe, E. Rojas, H.B. Pollard, Theoretical models of the ion channel structure of amyloid beta-protein, Biophysical journal 67 (1994) 2137-2145.

[11] X. Feng, J. Yang, Lysosomal Calcium in Neurodegeneration, Messenger (Los Angel) 5 (2016) 56-66.

[12] D.L. Groden, Z. Guan, B.T. Stokes, Determination of Fura-2 dissociation constants following adjustment of the apparent Ca-EGTA association constant for temperature and ionic strength, Cell Calcium 12 (1991) 279-287.

[13] G. Grynkiewicz, M. Poenie, R.Y. Tsien, A new generation of $\mathrm{Ca} 2+$ indicators with greatly improved fluorescence properties, The Journal of biological chemistry 260 (1985) 3440-3450.

[14] M.E. Guicciardi, M. Leist, G.J. Gores, Lysosomes in cell death, Oncogene 23 (2004) 2881-2890.

[15] T. Jakoš, A. Pišlar, A. Jewett, J. Kos, Cysteine Cathepsins in Tumor-Associated Immune Cells, 10 (2019).

[16] Z.S. Ji, R.D. Miranda, Y.M. Newhouse, K.H. Weisgraber, Y. Huang, R.W. Mahley, Apolipoprotein E4 potentiates amyloid beta peptide-induced lysosomal leakage and apoptosis in neuronal cells, The Journal of biological chemistry 277 (2002) 21821-21828.

[17] N. Kavčič, K. Pegan, B. Turk, Lysosomes in programmed cell death pathways: from initiators to amplifiers \%J Biological Chemistry, 398 (2017) 289.

[18] H. Lin, R. Bhatia, R. Lal, Amyloid beta protein forms ion channels: implications for Alzheimer's disease pathophysiology, FASEB journal : official publication of the Federation of American Societies for Experimental Biology 15 (2001) 2433-2444.

[19] H. Lin, Y.J. Zhu, R. Lal, Amyloid beta protein (1-40) forms calcium-permeable, Zn2+-sensitive channel in reconstituted lipid vesicles, Biochemistry 38 (1999) 11189-11196.

[20] M.-c.A. Lin, B.L. Kagan, Electrophysiologic properties of channels induced by Abeta25-35 in planar lipid bilayers, Peptides 23 (2002) 1215-1228.

[21] J.A. Mindell, Lysosomal acidification mechanisms, Annu Rev Physiol 74 (2012) 69-86.

[22] T. Mirzabekov, M.C. Lin, W.L. Yuan, P.J. Marshall, M. Carman, K. Tomaselli, I. Lieberburg, B.L. Kagan, Channel formation in planar lipid bilayers by a neurotoxic fragment of the beta-amyloid peptide, Biochemical and biophysical research communications 202 (1994) 1142-1148.

[23] H.B. Pollard, N. Arispe, E. Rojas, lon channel hypothesis for Alzheimer amyloid peptide 
neurotoxicity, Cellular and molecular neurobiology 15 (1995) 513-526.

[24] S.K. Rhee, A.P. Quist, R. Lal, Amyloid beta protein(1-42) forms calcium-permeable, Zn2+-sensitive channel, The Journal of biological chemistry 273 (1998) 13379-13382.

[25] M.A. Simmons, C.R. Schneider, Amyloid beta peptides act directly on single neurons, Neurosci Lett 150 (1993) 133-136.

[26] B. Turk, V. Stoka, J. Rozman-Pungercar, T. Cirman, G. Droga-Mazovec, K. Oresić, V. Turk, Apoptotic pathways: involvement of lysosomal proteases, Biological chemistry 383 (2002) 1035-1044.

[27] G.E. Villalpando Rodriguez, A. Torriglia, Calpain 1 induce lysosomal permeabilization by cleavage of lysosomal associated membrane protein 2, Biochimica et Biophysica Acta (BBA) - Molecular Cell Research 1833 (2013) 2244-2253.

[28] E. Wesén, G.D.M. Jeffries, M. Matson Dzebo, E.K. Esbjörner, Endocytic uptake of monomeric amyloid- $\beta$ peptides is clathrin- and dynamin-independent and results in selective accumulation of $A \beta(1-42)$ compared to $A \beta(1-40)$, Scientific reports 7 (2017) 2021-2021.

[29] A.J. Yang, D. Chandswangbhuvana, L. Margol, C.G. Glabe, Loss of endosomal/lysosomal membrane impermeability is an early event in amyloid Abeta1-42 pathogenesis, Journal of neuroscience research 52 (1998) 691-698.

[30] D.V. Zaretsky, M. Zaretskaia, Degradation Products of Amyloid Protein: Are They The Culprits?, Current Alzheimer research 17 (2020) 869-880.

[31] D.V. Zaretsky, M.V. Zaretskaia, Flow cytometry method to quantify the formation of beta-amyloid membrane ion channels, Biochimica et biophysica acta. Biomembranes (2020) 183506.

[32] D.V. Zaretsky, M.V. Zaretskaia, Mini-review: Amyloid degradation toxicity hypothesis of Alzheimer's disease, Neurosci Lett 756 (2021) 135959. 